\title{
Design Wireless Sensor Network and IoT for Smart City
}

\author{
Swarup Deshmukh ${ }^{1}$, Dr. C. N. Deshmukh ${ }^{2}$ \\ Department of Electronics \& Telecommunication ${ }^{1,2}$, PRMIT, Badnera Amravati, Maharashtra, India-444701,2 \\ Email: swarup.deshmukh21@gmail.com ${ }^{1}$,cndesh1968@gmail.com ${ }^{2}$
}

\begin{abstract}
Now a day's innovations in production of mobile computers and smart phones, sensors and sensor networks in connection with next generation mobile networks opened great opportunities for researchers and developers of various systems and application in the field of Smart Cities. Some typical applications including garbage monitoring, Humidity built-in sensors, water supply monitoring street light monitoring, and air pollution monitoring etc. require a smart gateway to provide high data rate, end-to-end connectivity is required with higher bandwidth. This paper proposes a novel model for Smart city concept using the IoT which has important benefits.
\end{abstract}

Index Terms- sensors, Smart Cities, Internet of Things

\section{INTRODUCTION}

Internet of Things can define as interconnection between people, animal or object that ability to exchange data over network without involving humanto-human or human-to-computer interaction. IOT offer various kinds of connectivity from devices, systems, and services that work within machine-to-machine communications and cover with applications, domains and protocols. Nowadays, there have many implementations of IoT devices, for instances, garbage monitoring, Humidity built-in sensors, water supply monitoring street light monitoring, air pollution monitoring etc. In current market the implementation of smart thermostat systems and washer/dryer for remote monitoring by using $\mathrm{Wi}-\mathrm{Fi}$.

In this work integrated system of Wi-Fi modem, IoT, Internet, Cameras and Sensor is introduced for efficient and economic garbage collection, Street Light Management, Water flow management, and Air pollution control in residential areas. The developed system provides improved for garbage collection time and waste amount at each location. We analyzed the solutions currently available for the implementation of IoT. By implementing this project we will avoid over flowing of garbage from the container in residential area which is previously either loaded manually or with the help of loaders in traditional trucks. It can automatically monitor the garbage bin \& send the image of garbage bin to collection truck. The technologies which are used in the proposed system are good enough to ensure the practical and perfect for solid garbage collection process monitoring and management for green environment. The system monitors the street light. System simply checks the intensity of ambient light to the threshold value if $\mathrm{ON}$ the street light. Otherwise OFF the street light.

\section{RELATED WORK}

Shanzhi Chen et. al. [1] proposed acting standard can hopefully balance desires from different parties, can open the door for future fundamental theory development, and can eventually stimulate/regulate IoT development. Recent years, Chinese government is pushing the development of the IoT. Following the Chinese 12th Five-Year Plan for IoT Development, China has accomplished a number of demonstration application projects such as the smart city and the intelligent transportation system in public IoT applications, intelligent coal mine, and the IOFs in industry applications.

Sanjana Prasad et. al. [2] deals with the design and implementation of Smart surveillance monitoring system using Raspberry pi and PIR sensor for mobile devices. It increases the usage of mobile technology to provide essential security to our homes and for other control applications. The proposed home security system captures information and transmits it via a $3 \mathrm{G}$ Dongle to a Smart phone using web application. Raspberry pi operates and controls motion detectors and video cameras for remote sensing and surveillance, streams live video and records it for future playback. It can also find the number of persons located with the help of the Infrared sensor. The cameras automatically initiate recording and the Raspberry pi device alerts the owner of the possible intrusion having a smart phone. Raspberry- Pi has two main components interacting with each other: one is the Web Application that executes on the mobile device's browser and server-side scripts that run in a cloud which will be operated by the Raspberry Pi Hardware tool component.

Adarsh Hegde et. al. [3] helps to automatically monitor the supply of water from the authorities and to get alert regarding it which helps in the proper utilization of it. This will help the masses to fill up their containers even in case of unusual timing of supply with automated procedures. Also the 


\section{E-ISSN: 2321-9637 \\ Available online at www.ijrat.org}

automation helps to reduce the wastage of water when containers are filled. Since in urban masses where water scarcity is quite common problem in summer, there is need to monitor the consumption of water used for domestic purposes. So that it is possible for judicial usage of water especially in the dry areas.

Akshay D. Deshmukh et. al. [4] intends to provide information using wireless sensor technology which comprises of raspberry pi, Arduino Nano, Zigbee, wireless sensor network(WSN) and sensors. Realization of data gathered by sensors based on embedded raspbian linux is displayed on Graphical User Interface (GUI). The system is developed using open source hardware raspberry pi and Zigbee which proves to be cost effective and having low power consumption. The sensors will gather the data of various environmental parameters and provide it to raspberry pi which act as a base station. Some sensors will directly process the data and provide it to the raspberry pi while some sensors will provide the data through Arduino Nano to raspberry pi using serial interface. The raspberry pi will then transmit the data using Zigbee and the processed data will be displayed on GUI through Zigbee that is on the receiver side.

Fabio Leccese et. al. [5] lamp posts used to light a crossroads and placed far from the city where the Internet signal does not arrive, has been re-designed combining all new technologies available on the market: LEDs for the lamps, PV panels for the power supply and an intelligent management. This last foresees an architecture that uses local sensors for intelligent lighting of the lamp, the storage of the functioning data, and their sharing by a local communication wireless mesh realized by ZigBee devices that send information to the coordinator lamp equipped with a RaspBerry-Pi card. The RaspBerry-Pi has been chosen for its low costs and for the possibility to drive also a WiMAX modem/router which allows to make the data system visible by a web site accessible by Internet also for areas very far from the city and not reached neither by the ADSL line nor by $3 \mathrm{G}$ signals.

Alexey Medvedev et. al. [6] proposed an advanced Decision Support System (DSS) for efficient waste collection in Smart Cities. The system incorporates a model for data sharing between truck drivers on real time in order to perform waste collection and dynamic route optimization. The system handles the case of ineffective waste collection in inaccessible areas within the Smart City. Surveillance cameras are incorporated for capturing the problematic areas and provide evidence to the authorities. The waste collection system aims to provide high quality of service to the citizens of a Smart City.

\section{PROPOSED METHOD}

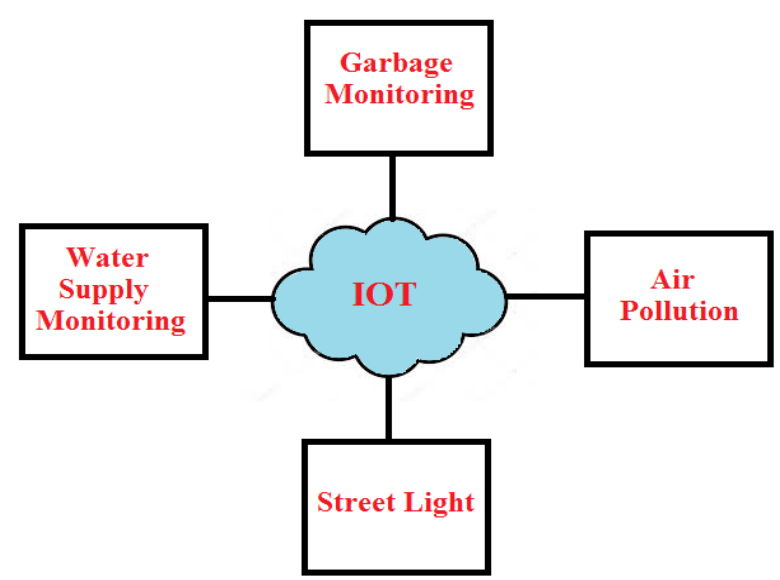

Figure 1: Basic Block diagram of proposed system

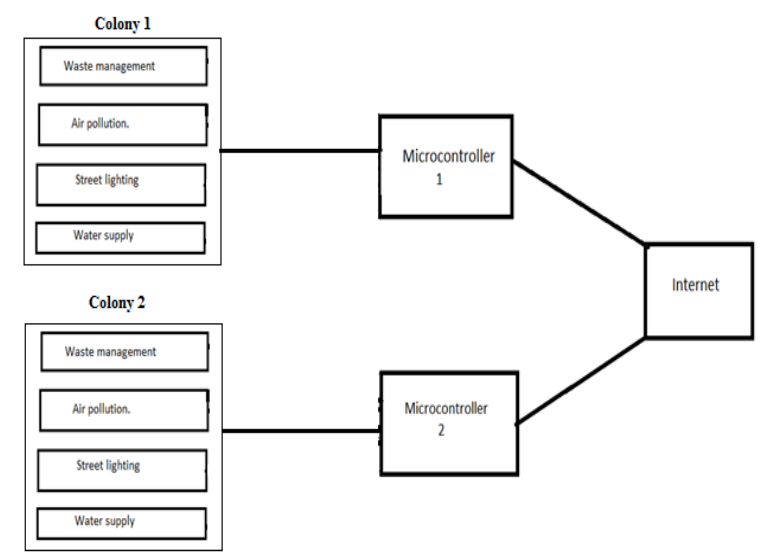

Figure 2: Block diagram of proposed system

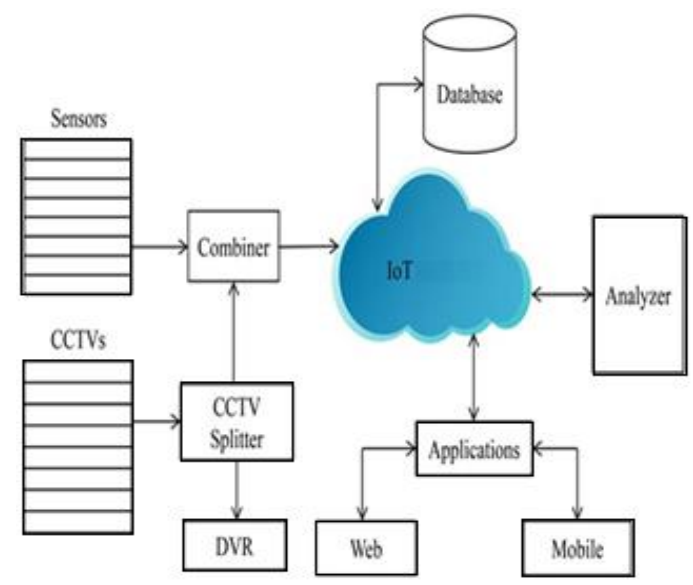

Figure 3: Flowchart of Proposed System 


\section{Available online at www.ijrat.org}

\section{EXPERIMENTAL SETUPS AND RESULT}

The proposed architecture is of IoT based for smart city. It include Garbage Monitoring or waste management, Water supply Monitoring, Street Light Monitoring, Air pollution Monitoring system shown in Fig. 1 is categorized into four modules

- Garbage Monitoring or waste management,

- Water supply Monitoring,

- Street Light Monitoring,

- Air pollution Monitoring

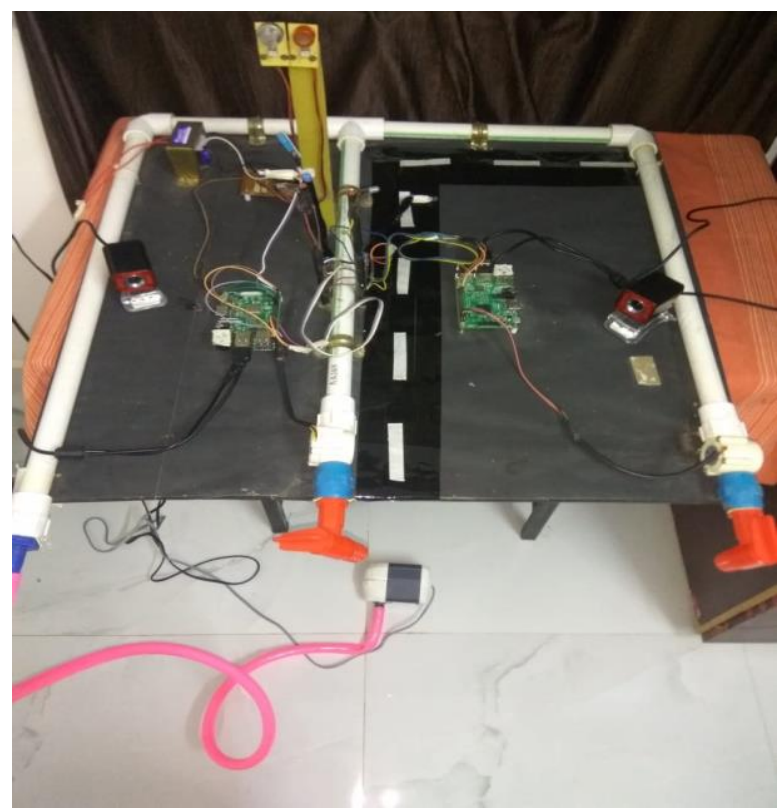

Figure 4: Experimental setup of proposed system

The proposed system contains chips. Chips consists Microcontroller along with various sensors like $\mathrm{CO} 2$ sensor, fog sensor, light sensor, humidity sensor, temperature sensor, water flow sensor and GSM network for wireless data sending and receiving between concentrator and PC. The data from the chips would get on a remote concentrator (PC), and the PC would also transfer the controlling action to the chip. According to the survey of variation in the intensity of light in the field area, an efficient programming would be done to ensure the least consumption of energy.

To the proposed system, we have used two raspberry pi, which are connected to each other through the WiFi.

One pin of each raspberry pi is connected to the ground. The pin no 19 and 23 of each raspberry pi are connected to the street light for which we have used two LED for each.

Pin no 2 of raspberry pi 1 and raspberry pi 2 is connected to water flow. The proposed system is working on $+5 \mathrm{~V}$ power supply.

\section{FLOWCHART OF PROPOSED SYSTEM}

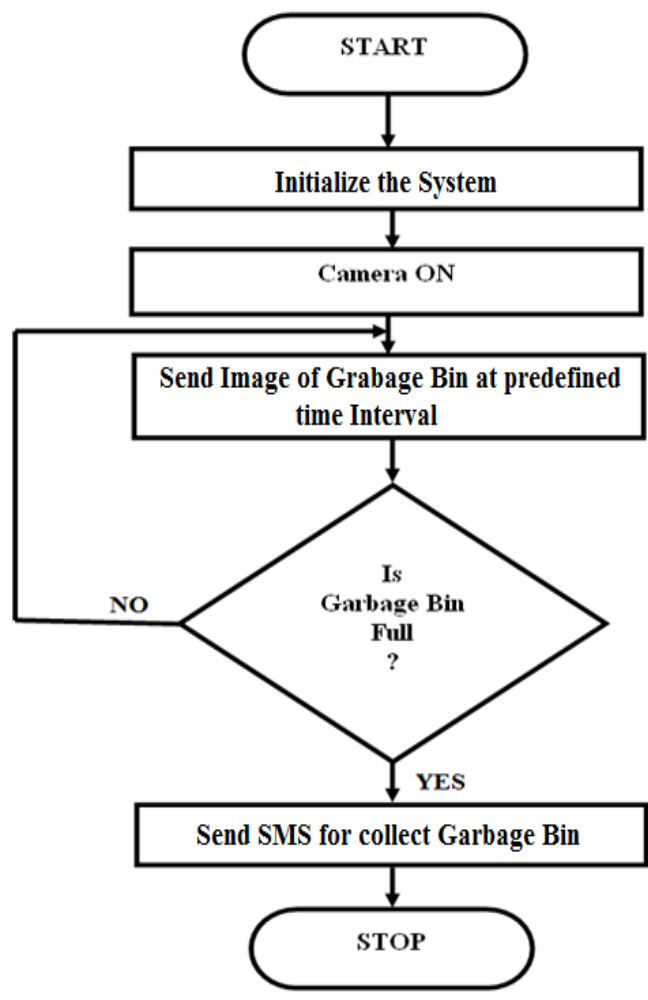

Figure 5: Flowcharts for Waste management

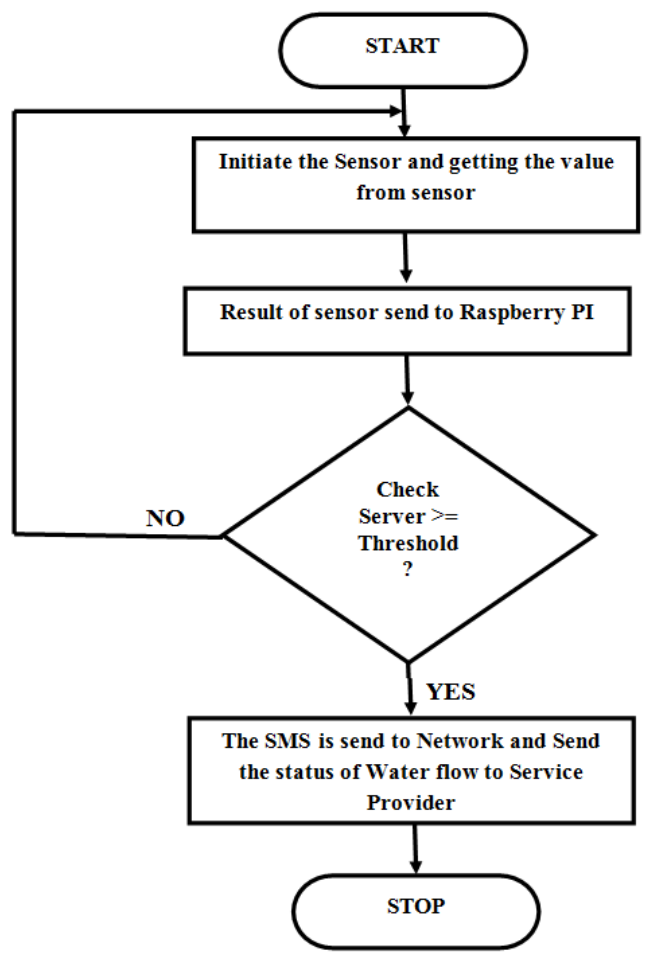

Figure 6: flowchart for Water supply Monitoring 


\section{Available online at www.ijrat.org}

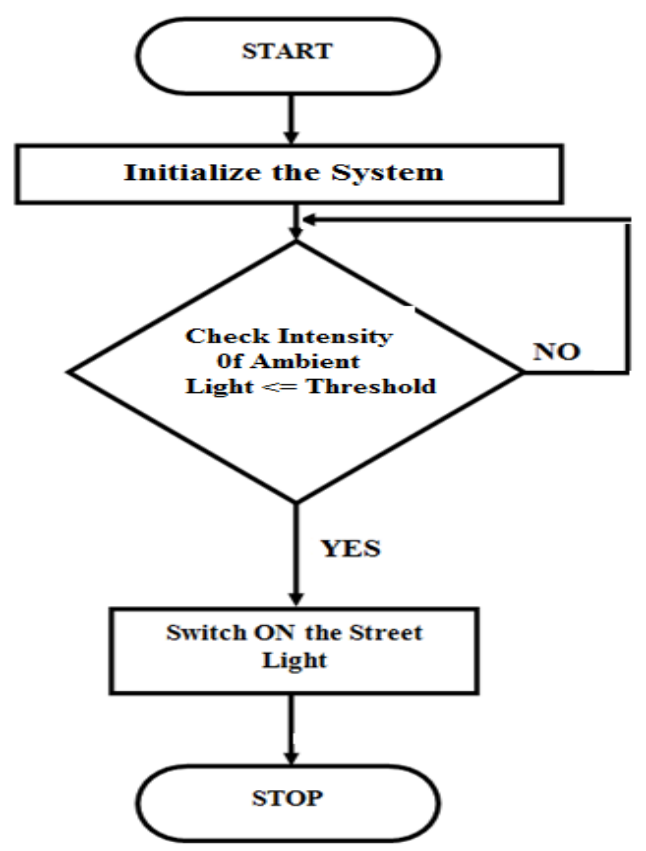

Figure 7: Flowchart for Street Light Monitoring

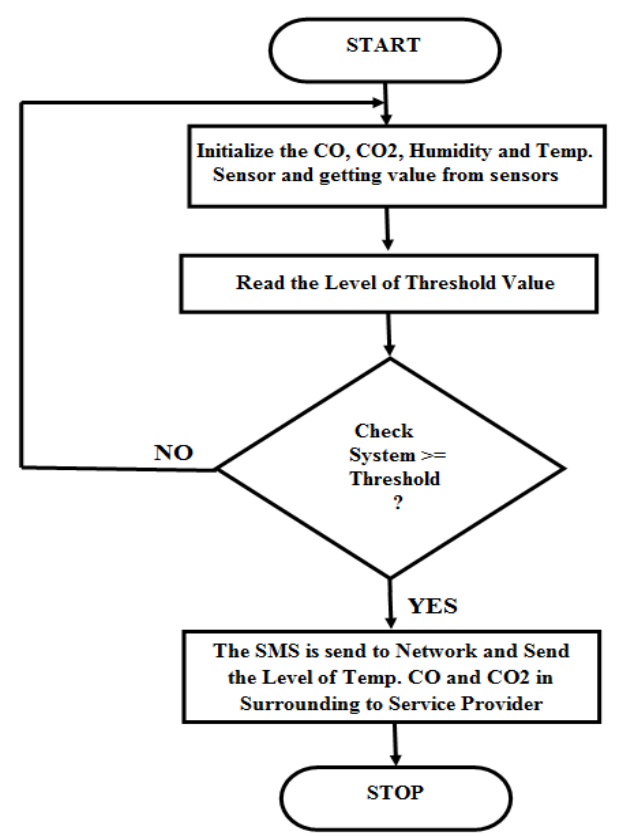

Figure 8: Flowchart for Air pollution Monitoring

\section{CONCLUSION}

These paper designs a low cost Wireless Sensor Network monitoring system including sensor node, Raspberry $\mathrm{Pi}$ as base station. The system designed here is easy to deploy having low cost, low power consumption, more reliable and simple to handle. One major advantage provided by the system is that, it has node of Wireless Sensor Network, database server and web server into a single compact credit sized microcomputer Raspberry Pi. In addition, this system allows us to integrate other hardware components with the Raspberry $\mathrm{Pi}$ as a credit size microcomputer. Considering the system ability more sensing node can be added for application specific designing. The detail design and measurement result demonstrated shows the usefulness of this system.

\section{REFERENCES}

[1] Dongdong Hou, Weiming Zhang, Kejiang Chen, Sian-Jheng Shanzhi Chen, Hui Xu, Dake Liu, Bo $\mathrm{Hu}$, and Hucheng Wang, "A Vision of IoT: Applications, Challenges, and Opportunities With China Perspective", IEEE INTERNET OF THINGS JOURNAL, VOL. 1, NO. 4, AUGUST 2014

[2] Sanjana Prasad1, P.Mahalakshmi2, A.John Clement Sunder3, R.Swathi4, "Smart Surveillance Monitoring System Using Raspberry PI and PIR Sensor", International Journal of Computer Science and Information Technologies, Vol. 5 (6) , 2014, 7107-7109.

[3] Adarsh Hegde, Gopi Kiran T S , Deepthi D , T N Nagabhushan, S P Shiva Prakash and Anand Raj S Ulle, "Automated Water flow Control System", National Conference on Product Design (NCPD 2016), July 2016.

[4] Akshay D. Deshmukh, Ulhas B. Shinde, "A Low Cost Environment Monitoring System Using Raspberry Pi and Arduino with Zigbee", IEEE, International Conference on Inventive Computation Technologies (ICICT), 26-27 Aug. 2016.

[5] Fabio Leccese, Marco Cagnetti and Daniele Trinca, "A Smart City Application: A Fully Controlled Street Lighting Isle Based on Raspberry-Pi Card, a ZigBee Sensor Network and WiMAX", Sensors 2014, 14, 24408-24424; doi:10.3390/s141224408.

[6] Alexey Medvedev, Petr Fedchenkov, Arkady Zaslavsky, Theodoros Anagnostopoulos, Sergey Khoruzhnikov, "Waste management as an IoT enabled service in Smart Cities", Springer-Verlag Berlin Heidelberg 2015.

[7] Cheah Wai Zhao, Jayanand Jegatheesan, Son Chee Loon. "Exploring IOT Application Using Raspberry Pi", International Journal of Computer Networks and Applications, Volume 2, Issue 1, January - February 2015.

[8] C. Balasubramaniyan and D. Manivannan, "IoT Enabled Air Quality Monitoring System (AQMS), using Raspberry Pi", Indian Journal of Science and Technology, Vol 9(39), DOI: 10.17485/ijst/2016/v9i39/90414, October 2016. 